Claremont Colleges

Scholarship@ Claremont

All HMC Faculty Publications and Research

HMC Faculty Scholarship

$1-1-1992$

\title{
Optical Rectification at Semiconductor Surfaces
}

Shun Lien Chuang

University of Illinois at Urbana-Champaign

Stefan Schmitt-Rink

AT\&T Bell Laboratories

Benjamin I. Greene

AT\&T Bell Laboratories

Peter N. Saeta

Harvey Mudd College

Anthony F.J. Levi

AT $\mathcal{T} T$ Bell Laboratories

\section{Recommended Citation}

"Optical rectifcation at semiconductor surfaces," S. L. Chuang, S. Schmitt-Rink, B. I. Greene, P. N. Saeta, and A. F. J. Levi, Phys. Rev. Lett. 68, 102 (1992).

This Article is brought to you for free and open access by the HMC Faculty Scholarship at Scholarship @ Claremont. It has been accepted for inclusion in All HMC Faculty Publications and Research by an authorized administrator of Scholarship @ Claremont. For more information, please contact scholarship@cuc.claremont.edu. 


\title{
Optical Rectification at Semiconductor Surfaces
}

\author{
Shun Lien Chuang \\ Department of Electrical and Computer Engineering, University of Illinois at Urbana-Champaign, Urbana, Illinois 61801
}

Stefan Schmitt-Rink, (a) Benjamin I. Greene, Peter N. Saeta, and Anthony F. J. Levi

AT\& T Bell Laboratories, Murray Hill, New Jersey 07974

(Received 16 July 1991)

\begin{abstract}
We show that far-infrared radiation can be generated in the depletion field near semiconductor surfaces via the inverse Franz-Keldysh effect or electric-field-induced optical rectification. This mechanism is conceptually different from those previously proposed and accounts for many recent experimental observations.
\end{abstract}

PACS numbers: $78.20 . \mathrm{Jq}, 42.65 . \mathrm{Bp}, 73.25 .+\mathrm{i}$

A significant body of work dating back to the early days of pulsed lasers has explored the generation of farinfrared (FIR) radiation using visible-light sources and electro-optic materials. In such materials, the nonlinear interaction between two optical fields produces a static or slowly varying electric field at the beat frequency, with an intensity determined by the instantaneous intensity of the optical fields. This phenomenon has been designated as the "inverse electro-optic effect," "optical rectification," or, more generally, "difference frequency mixing" [1]. A number of picosecond time scale demonstrations of this effect have appeared in the literature [2]. More recently, the generation and detection of picosecond and subpicosecond FIR transients has taken a new direction, utilizing photoconductive media coupled to antenna structures [3]. In these experiments the FIR generation process has been explained as Hertzian radiation from time-varying electric currents.

Very recently, it has been shown experimentally that ultrashort pulses of FIR radiation can also be generated from ultrashort optical pulses incident on a semiconductor surface $[4,5]$. This observation opens up new and exciting possibilities for terahertz spectroscopy and characterization of electronic properties of semiconductor surfaces. These studies have culminated in the observation of FIR transients as short as $120 \mathrm{fs}$ [5]. The commonly accepted explanation for the effect has remained in the domain of "current surges" occurring in the surface depletion field [4]. In the present work, however, we wish to reexamine this most recent phenomenon in the original context of optical rectification and second-order optical nonlinearities. Our model quantitatively takes into account both the above band-gap (resonant) nature of the excitation process as well as the presence of a surface depletion field. In so doing, we find that we can satisfactorily account for many key experimental observations, most importantly the absolute intensity of the detected light and its dependence on crystallographic orientation.

Figure 1 shows a simple representation of the energyband extrema of a semiconductor near the surface. In a conventional transport picture one assumes that electronhole (e-h) pairs are created by vertical transitions in real space within the penetration depth of the incident optical field. Subsequently, as indicated by the solid arrows in Fig. 1, electrons are accelerated by the surface depletion field, while holes remain confined to the surface. The resulting current surge $\partial J / \partial t$ then gives rise to FIR radiation, where $J$ is proportional [4] to the photoelectron population $n$ and material mobility $\mu$. However, as indicated by the dashed arrow in Fig. 1, there exists an alternative approach to the problem, based on nonvertical transitions in real space. Because of spatial separation of final electron and hole states, this process leads to an instantaneous FIR polarization $P_{0}$ whose second time derivative $\partial^{2} P_{0} / \partial t^{2}$ determines the radiated signal. This stationary-state picture also underlies some theoretical descriptions of the Franz-Keldysh effect, i.e., the modification of optical properties by a static electric field [6].

In lowest order in the optical field $E_{\omega}$ and neglecting nonlocal effects, the FIR (static) polarization $P_{0}$ induced perpendicular to the surface may be written as

$$
P_{0}=\chi^{(2)}(0 ;-\omega, \omega)\left|E_{\omega}\right|^{2},
$$

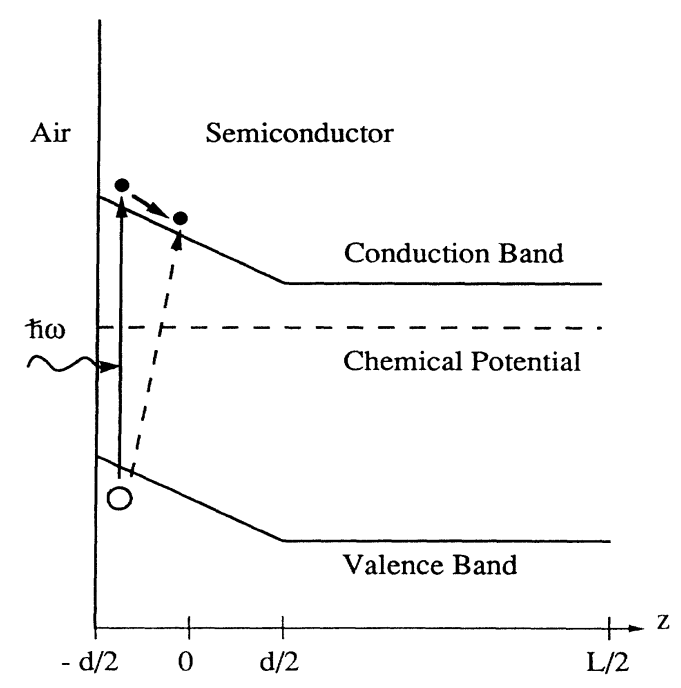

FIG. 1. Energy-band extrema near a semiconductor surface. 
where $\chi^{(2)}$ is the second-order nonlinear optical susceptibility [7]. Usually, the latter quantity is strictly equal to zero in systems with inversion symmetry, such as semiconductors with diamond structure. However, the surface depletion field $F$ breaks this symmetry and makes $\chi^{(2)}$ nonzero over the depletion width. For the simple two-band semiconductor model illustrated in Fig. 1, we find in the rotating-wave and effective-mass approximations

$$
\chi^{(2)}(0 ;-\omega, \omega)=-\frac{1}{V} \sum_{n m \mathbf{k} s} \frac{\mu_{n m}}{\varepsilon_{\mathrm{e} n \mathbf{k}}+\varepsilon_{\mathrm{h} m \mathbf{k}}-\omega-i \gamma}\left(\sum_{n^{\prime}} \frac{\left\langle n^{\prime}\left|e z_{\mathrm{e}}\right| n\right\rangle \mu_{n^{\prime} m}^{*}}{\varepsilon_{\mathrm{e} n^{\prime} k}+\varepsilon_{\mathrm{h} m \mathbf{k}}+\omega+i \gamma}-\sum_{m^{\prime}} \frac{\left\langle m\left|e z_{\mathrm{h}}\right| m^{\prime}\right\rangle \mu_{n m^{\prime}}^{*}}{\varepsilon_{\mathrm{e} n \mathbf{k}}+\varepsilon_{\mathrm{h} m^{\prime} \mathbf{k}}-\omega+i \gamma}\right)
$$

Here, $\hbar=1, V$ denotes the crystal volume, $\gamma$ the dephasing rate, $s$ the spin, $\mathbf{k}$ the momentum parallel to the surface $(x-y$ direction), and $n$ the electron and hole eigenstates perpendicular to the surface ( $z$ direction). $\left\langle n^{\prime}\left|e z_{\mathrm{e}}\right| n\right\rangle,\left\langle m\left|e z_{\mathrm{h}}\right| m^{\prime}\right\rangle$, and $\mu_{n m}$ are the intraband and interband dipole matrix elements, respectively. In our calculation, infinite barriers are assumed at the air/semiconductor interface $z=-d / 2$ and at $z=L / 2$, as shown in Fig. 1. As $L \rightarrow \infty$, the bulk (half-space) limit is obtained.

The most striking evidence for the $\chi^{(2)}$ process is a strong modulation of the detected FIR intensity as the sample is rotated about its surface normal. Figure 2(a) shows the observed FIR intensity (triangles) from an InP(100) surface as a function of azimuthal angle as well as a fit by a theoretical curve of the form $c_{1}+c_{2} \cos 2 \phi$, where $\phi$ is measured from the (011) direction and the

coefficients $c_{1}$ and $c_{2}$ depend on the angle of incidence and the linear optical response. The calculation takes into account the anisotropy of $\chi^{(2)}$ in an approach similar to that in Refs. [7,8]. Similarly, Fig. 2(b) shows the azimuthal dependence of the FIR intensity from an InP(111) surface, which is well described by the theoretical expression

$$
\begin{gathered}
c_{1}^{\prime}+c_{2}^{\prime}[\alpha \cos \phi-1][\alpha \cos (\phi-2 \pi / 3)-1] \\
\times[\alpha \cos (\phi-4 \pi / 3)-1],
\end{gathered}
$$

where $\alpha=\sqrt{2} \cot \theta_{t}$, and $\theta_{t}$ is the transmission angle of the $p$-polarized light. The observed strong modulation of FIR intensity, which arises from the anisotropy of $\chi^{(2)}$, is difficult to explain in the current surge model.

For small surface depletion fields, we expand the dipole matrix elements in Eq. (2) in powers of $F$. Retaining only the leading term, we find

$$
\begin{aligned}
\chi^{(2)}(0 ;-\omega, \omega)=\frac{\left|\mu_{0}\right|^{2}}{V} \sum_{n m \mathbf{k} . s} & \frac{1}{\varepsilon_{\mathrm{e} n \mathbf{k}}+\varepsilon_{\mathrm{h} m \mathbf{k}}-\omega+i \gamma}\left(\frac{\left\langle n\left|e z_{\mathrm{e}}\right| m\right\rangle_{0}}{\varepsilon_{\mathrm{e} m \mathbf{k}}+\varepsilon_{\mathrm{h} m \mathbf{k}}-\omega-i \gamma}-\frac{\left\langle n\left|e z_{\mathrm{h}}\right| m\right\rangle_{0}}{\varepsilon_{\mathrm{e} n \mathbf{k}}+\varepsilon_{\mathrm{h} n \mathbf{k}}-\omega-i \gamma}\right) \\
& \times\left(\frac{\left\langle m \mid e F z_{\mathrm{e}}<n\right\rangle_{0}}{\varepsilon_{\mathrm{e} m \mathbf{k}}+\varepsilon_{\mathrm{h} m \mathbf{k}}-\omega+i \gamma}-\frac{\left\langle m\left|e F z_{\mathrm{h}}{ }^{<}\right| n\right\rangle_{0}}{\varepsilon_{\mathrm{e} n \mathbf{k}}+\varepsilon_{\mathrm{h} n \mathbf{k}}-\omega+i \gamma}\right)+\text { c.c. },
\end{aligned}
$$

where $z_{\mathrm{e}, \mathrm{h}}=\Theta\left(d / 2-z_{\mathrm{e}, \mathrm{h}}\right)\left(z_{\mathrm{e}, \mathrm{h}}-d / 2\right), \mu_{0}$ is the bulk interband dipole matrix element, and $\Theta$ is the Heaviside step function. This result may also be written in the intuitive form

$$
\chi^{(2)}(0 ;-\omega, \omega)=\chi^{(3)}(0 ; 0,-\omega, \omega) F
$$

which shows that the effect is entirely induced by the symmetry-breaking surface depletion field $F$.

Equation (3) is our main result. It generalizes to arbitrary photon energies $\omega$ and nonzero dephasing rate $\gamma$ the results of Ref. [9], where below band gap (nonresonant) excitation of an externally biased semiconductor has been considered. In this case, by using the permutation relations for nonlinear optical susceptibilities [7], $\chi^{(3)}(0 ; 0$, $-\omega, \omega)$ may be directly related to $\chi^{(3)}(-\omega ; \omega, 0,0)$ describing quadratic electrorefraction, i.e., the leading changes in refractive index due to a static electric field [9]. In the present case, the relationship between Eq. (3) and changes in optical properties due to a surface depletion field is more complicated. We note, however, that large effects have been observed using various kinds of modulation spectroscopy [10] and hence there exists an equally large nonlinear optical susceptibility describing surface depletion field-induced optical rectification.

We have evaluated Eq. (3) for GaAs parameters: band-gap energy $E_{g}=1.425 \mathrm{eV}$, spin split-off energy $\Delta=0.33 \mathrm{eV}$, electron effective mass $m_{\mathrm{e}}=0.067 m_{0}$, and hole effective mass $m_{\mathrm{h}}=0.34 m_{0}$. Figure 3(a) shows the third-order nonlinear optical susceptibility $\chi^{(3)}(0 ; 0,-\omega$, $\omega)$ versus photon energy $\omega$ for a dephasing rate $\gamma=5$ $\mathrm{meV}$ and (i) a surface depletion field $(d=200 \AA, L$ $=1800 \AA$ ), (ii) an externally biased thin film or quantum well $(d=L=200$ and $1000 \AA)$, (iii) an externally biased bulk sample $(d=L=\infty)$. In all cases $\chi^{(3)}$ shows different behavior for excitation below and above the band gap $E_{g}$. For $\omega<E_{g}, \chi^{(3)}$ drops rapidly, following a power-law behavior. This regime has been discussed previously and designated as virtual photoconductivity [9]. For $\omega>E_{g}$, on the other hand, $\chi^{(3)}$ follows more or less the linear absorption, as expected for real photoconductivity. We note, however, that our expression [Eq. (3)] for $\chi^{(3)}$ also contains contributions due to the optically induced interband polarization and hence differs from any consideration based on population effects alone [4]. These additional coherent contributions are particularly 

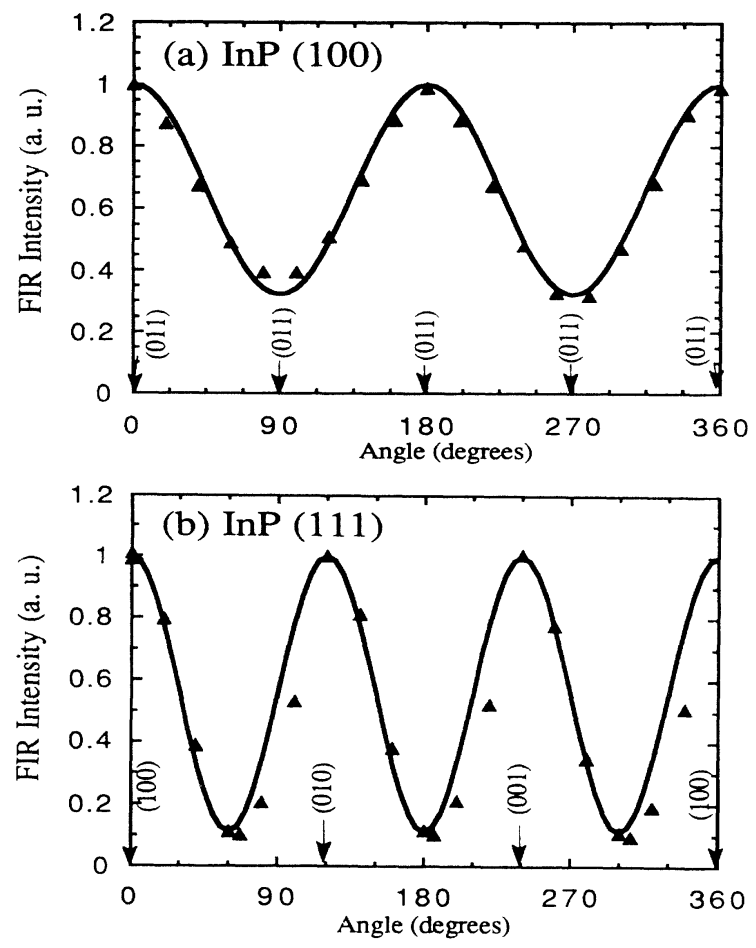

FIG. 2. Crystal orientation dependence of the femtosecond electromagnetic signal generated from 100-fs 2-eV optical pulses as a function of the azimuthal angle $\phi$ for an InP sample grown along (a) the (100) direction and (b) the (111) direction. The solid triangles are the experimental data. Theoretical results are shown as solid curves.

important on the picosecond and subpicosecond time scales. In any case, Fig. 3(a) is consistent with the experimental result that no detectable FIR radiation is obtained from $\mathrm{ZnSe}\left(E_{g}=2.4 \mathrm{eV}\right)$ and $\mathrm{GaP}\left(E_{g}=2.2 \mathrm{eV}\right)$ samples when excited well below the band gap at $\omega=2$ eV [4].

The optical- and electric-field dependences of the FIR radiation follow directly from Eqs. (1) and (3b) and are also consistent with experiments [4]. The induced FIR polarization scales linearly with optical intensity and surface depletion field $F$. It thus has a different sign in $n$ type and $p$-type samples [4]. For very high optical intensities, we expect the optical intensity dependence of the induced FIR polarization to saturate to a sublinear behavior due to (i) saturation of interband transitions [9] and, more importantly, (ii) screening of the low-frequency FIR response. Indeed, this saturation has been observed in recent ultrashort pulse experiments [5].

Figure 3(b) shows that for fixed $\omega(\omega=2 \mathrm{eV})$ and the above band-gap excitation $\chi^{(3)}$ decreases with increasing dephasing rate $\gamma$. This is again consistent with experiments in which a marked decrease of the FIR signal with increasing temperature has been found [4]. (We note, however, that the surface depletion field $F$ and width $d$ also depend weakly on temperature [11].) Since the de-
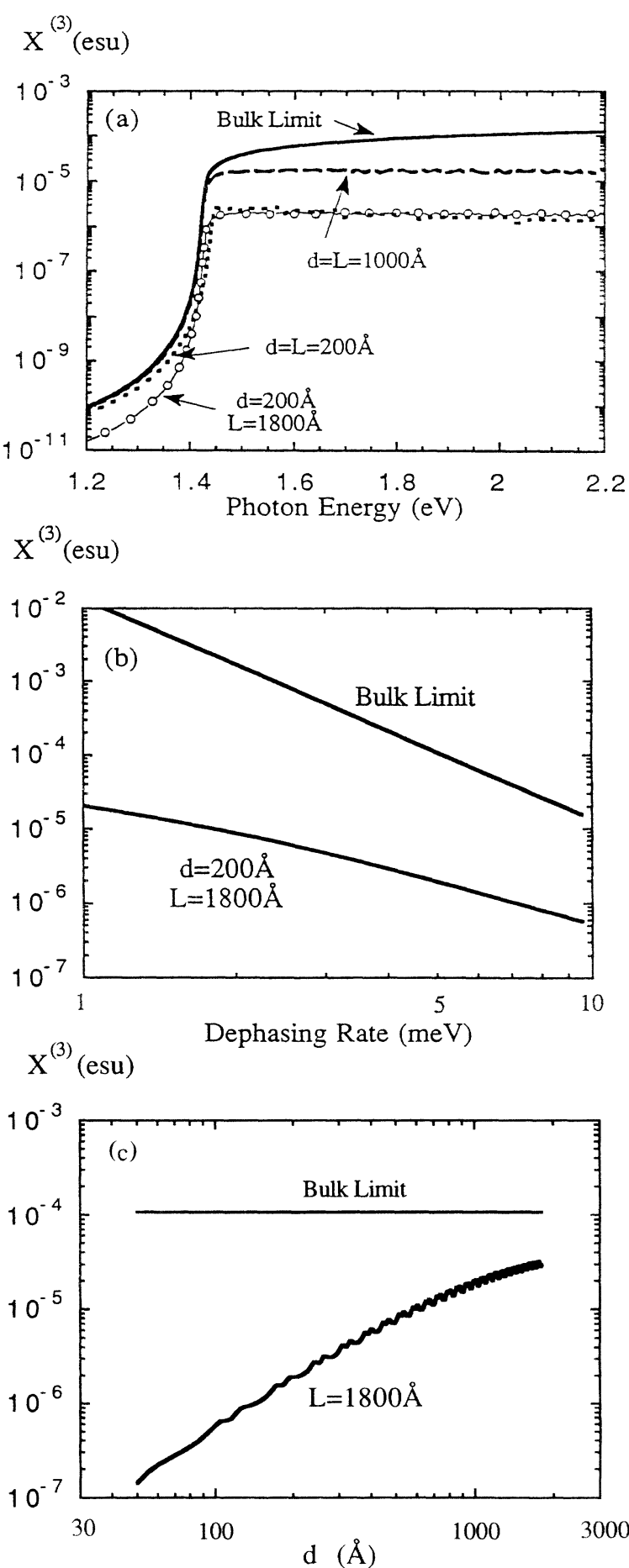

FIG. 3. Third-order nonlinear optical susceptibility $\chi^{(3)}(0$; $0,-\omega, \omega)$ of GaAs vs (a) photon energy $\omega$, (b) dephasing rate $\gamma$, and (c) depletion width $d$.

phasing time is approximately proportional to the mobility, this result confirms the picture that the induced FIR polarization scales with mobility [4].

Figure 3(c) shows $\chi^{(3)}$ vs $d$ for fixed $\gamma$ and $\omega(\gamma=5$ 
meV and $\omega=2 \mathrm{eV}$ ). The dependence on depletion width $d$ follows approximately a linear behavior for $d>300 \AA$ (or doping $n<10^{18} \mathrm{~cm}^{-3}$ ) [10] and a quadratic behavior for $d<300 \AA$. Using the well-known doping dependence of the surface depletion field and width $F \sim n^{0.5}$ and $d \sim n^{-0.5}$, respectively [11], we find for the doping dependence of the radiated FIR field $E_{0} \sim P_{0} d \sim F d^{2} \sim n^{-0.5}$ for $d>300 \AA$, which is again in good agreement with the experimental result [4] $E_{0} \sim n^{-0.4}$. Using a dipole model similar to that used by Shen [12], we also find that the radiated FIR fields in reflection and transmission have opposite signs, again in agreement with observation [4].

We point out that our calculated absolute values of $\chi^{(3)}$ (Fig. 2) are also quite consistent with measured efficiencies (in the unsaturated regime) for FIR generation at semiconductor surfaces. Specifically, depending on the detailed doping parameters, calculated values of $\chi^{(3)}$ between $10^{-6}$ and $10^{-5}$ esu lead to FIR field strengths on the order of $10 \mathrm{~V} / \mathrm{cm}$, assuming an incident optical power density of approximately $10^{3} \mathrm{~W} / \mathrm{cm}^{2}$.

In conclusion, we propose that a model based on optical rectification at semiconductor surfaces is sufficient to explain all the major experimental observations made to date. In particular, our model provides a simple explanation of the strong dependence of FIR generation on crystallographic orientation of the sample surface. Furthermore, we suggest that the present mechanism should also give rise to second-harmonic $(\mathrm{SH})$ generation. We do expect some differences between FIR and $\mathrm{SH}$ generation, however, due to the different screening of the lowfrequency FIR response and the high-frequency $\mathrm{SH}$ response.

We thank Wayne Knox, Martin Nuss, and Eli Yablonovitch for useful discussions. The work of S.L.C. was supported by the Office of Naval Research (Grant No. N00014-90-J-1821). Supercomputer time was provided by the National Center for Supercomputing Applications at the University of Illinois. (a) Present address: Department of Physics and Materials Sciences Center, Philipps University, 3550 Marburg, Federal Republic of Germany.

[1] M. Bass, P. A. Franken, J. F. Ward, and G. Weinreich, Phys. Rev. Lett. 9, 446 (1962).

[2] J. R. Morris and Y. R. Shen, Opt. Commun. 3, 81 (1971); D. H. Auston, K. P. Cheung, J. A. Valdmanis, and D. A. Kleinman, Phys. Rev. Lett. 53, 1555 (1984).

[3] P. R. Smith, D. H. Auston, and M. C. Nuss, IEEE J. Quantum Electron. 24, 255 (1988); M. van Exter, Ch. Fattinger, and D. Grischkowsky, Appl. Phys. Lett. 55, 337 (1989).

[4] X. C. Zhang, B. B. Hu, J. T. Darrow, and D. H. Auston, Appl. Phys. Lett. 56, 1011 (1990); X. C. Zhang, J. T. Darrow, B. B. Hu, D. H. Auston, M. T. Schmidt, P. Tham, and E. S. Yang, Appl. Phys. Lett. 56, 2228 (1990); X. C. Zhang and D. H. Auston, J. Appl. Phys. (to be published).

[5] B. I. Greene, J. F. Federici, D. R. Dykaar, A. F. J. Levi, and L. N. Pfeiffer, Opt. Lett. 16, 48 (1991); B. I. Greene, J. F. Federici, D. R. Dykaar, R. R. Jones, and P. H. Bucksbaum, Appl. Phys. Lett. 59, 893 (1991).

[6] D. A. B. Miller, D. S. Chemla, and S. Schmitt-Rink, Phys. Rev. B 33, 6976 (1986).

[7] N. Bloembergen, Nonlinear Optics (Benjamin, New York, 1965).

[8] H. W. K. Tom, T. F. Heinz, and Y. R. Shen, Phys. Rev. Lett. 51, 1983 (1983).

[9] M. Yamanishi, Phys. Rev. Lett. 59, 1014 (1987); D. S. Chemla, D. A. B. Miller, and S. Schmitt-Rink, Phys. Rev. Lett. 59, 1018 (1987); E. Yablonovitch, J. P. Heritage, D. E. Aspnes, and Y. Yafet, Phys. Rev. Lett. 63, 976 (1989); Y. Yafet and E. Yablonovitch, Phys. Rev. B 43, 12480 (1991)

[10] H. Shen and F. H. Pollak, Phys. Rev. B 42, 7097 (1990); Y. Yin, D. Yan, F. H. Pollak, G. D. Pettit, and J. M. Woodall, Phys. Rev. B 43, 12138 (1991).

[11] S. M. Sze, Physics of Semiconductor Devices (Wiley, New York, 1981).

[12] Y. R. Shen, Annu. Rev. Phys. Chem. 40, 327 (1989). 